\title{
Investigation of the Class of the Rational Difference Equations
}

\author{
Elmetwally M. Elabbasy¹, Osama Moaaz ${ }^{1}$, Shaimaa Alsaeed ${ }^{2}$ \\ ${ }^{1}$ Department of Mathematics, Faculty of Science, Mansoura University, Mansoura, Egypt \\ ${ }^{2}$ Department of Mathematics, The Faculty of Education, Al al-Bayt University, Mafraq, Jordan \\ Email: emelabbasy@mans.edu.eg,o_moaaz@mans.edu.eg, shaimaa.alsaeed@yahoo.com
}

How to cite this paper: Elabbasy, E.M., Moaaz, O. and Alsaeed, Sh. (2017) Investigation of the Class of the Rational Difference Equations. Applied Mathematics, 8, 1464-1472.

https://doi.org/10.4236/am.2017.810107

Received: July 19, 2017

Accepted: October 28, 2017

Published: October 31, 2017

Copyright (c) 2017 by authors and Scientific Research Publishing Inc. This work is licensed under the Creative Commons Attribution International License (CC BY 4.0).

http://creativecommons.org/licenses/by/4.0/

\begin{abstract}
This paper is concerned with asymptotic behavior of the solution of a new class of rational Difference Equations. We consider the local and global stability of the solution. Moreover we investigate the new periodic character (periodic two) of solutions of these equations. Finally, we give some interesting counter examples in order to verify our strong results.
\end{abstract}

\section{Keywords}

Difference Equation, Stability, Periodicity, Boundedness

\section{Introduction}

The objective of this work is to investigate the asymptotic behavior of the solutions of the following difference equation

$$
\omega_{n+1}=a+\frac{b_{0} \omega_{n-1}}{c_{1} \omega_{n}+c_{2} \omega_{n-1}}+\frac{b_{1} \omega_{n-3}}{c_{3} \omega_{n-2}+c_{4} \omega_{n-3}}, n=0,1,2, \cdots
$$

where $a, b_{0}, b_{1}, c_{1}, c_{2}, c_{3}$ and $c_{4} \in[0, \infty)$ and the initial conditions $\omega_{-3}, \cdots, \omega_{-1}$ and $\omega_{0}$ are arbitrary positive real numbers.

In recent years, there are a great interest in studying the rational difference equations. These equations describe real life situations in queuing theory, stochastic time series, combinatorial analysis, electrical network, number theory, genetics in biology, psychology, probability theory, statistical problems, economics, etc. The study of rational difference equations of high order (greater than one) is a big challenge. However, there have not been any effective general methods to deal with the global behavior of rational difference equations of order greater than one. Therefore, the study of such equations is so interesting. There has been a lot of work concerning the global asymptotic of solutions of ra- 
tional difference equations [1]-[21].

In fact, there has been a lot of interest in studying the behavior of the nonlinear difference equation of the form

$$
x_{n+1}=\alpha+\frac{x_{n-1}}{x_{n}} .
$$

For multiple delay and order, see [3] [18] [19] [20] and their references for more results of this equation. In Theorems 4.7.1-4.7.5 in [12], Kulenovic investigated the asymptotic behavior of the solutions of the equation

$$
x_{n+1}=\frac{\gamma x_{n-1}}{B x_{n}+C x_{n-1}} .
$$

Metwally et al. in [14] established a global convergence result and then apply it to show that under appropriate hypotheses every positive solution of the difference equation

$$
x_{n+1}=\sum_{i=0}^{m} \frac{A_{i}}{x_{n-2 i}},
$$

where $A_{i} \in[0, \infty)$ for $i=0,1, \cdots, m$. In [8], Elsayed studied the periodicity, the boundedness, and the global stability of the positive solution of the difference equation

$$
x_{n+1}=\alpha+\beta \frac{x_{n}}{x_{n-1}}+\gamma \frac{x_{n-1}}{x_{n}},
$$

where the parameters $\alpha, \beta$ and $\gamma$ are positive real numbers and the initial conditions $x_{-1}, x_{0}$ are positive real numbers. Recently, Moaaz et al. [15] investigated some qualitative behavior of the following nonlinear difference equations

$$
x_{n+1}=\alpha+\beta \frac{x_{n-l}}{x_{n-k}}+\gamma \frac{x_{n-l}}{x_{n-s}},
$$

where the initial conditions $x_{-r}, x_{-r+1}, \cdots, x_{0}$ such that $r=\max \{l, k, s\}$ are arbitrary real numbers and $\alpha, \beta$ and $\gamma$ are positive constants.

In this paper, in section 2 , we state the sufficient condition for the asymptotic stability of Equation (1). Next, in section 3, we study the existence of periodic solutions of Equation (1). Finally, we study the boundedness nature of the solutions of Equation (1). Some numerical examples will be given to explicate our results.

During this study, we will need to many of the basic concepts. Before anything, the concept of equilibrium point is essential in the study of the dynamics of any physical system. A point $\bar{\omega}$ in the domain of the function $\Phi$ is called an equilibrium point of the equation

$$
\omega_{n+1}=\Phi\left(\omega_{n}, \omega_{n-1}, \cdots, \omega_{n-k}\right), n=0,1,2, \cdots
$$

if $\bar{\omega}$ is a fixed point of $\Phi[\Phi(\bar{\omega}, \bar{\omega}, \cdots, \bar{\omega})=\bar{\omega}]$. For a stability of equilibrium point, equilibrium point $\bar{\omega}$ of Equation (4) is said to be locally stable if for all $\varepsilon>0$ there exists $\delta>0$ such that, if $\omega_{-v} \in(0, \infty)$ for $v=0,1, \cdots, k$ with 
$\sum_{i=0}^{k}\left|\omega_{-i}-\bar{\omega}\right|<\delta$. As well, $\bar{\omega}$ is said to be locally asymptotically stable if it is locally stable and there exists $\gamma>0$ such that, if $\omega_{-v} \in(0, \infty)$ for $v=0,1, \cdots, k$ with $\sum_{i=0}^{k}\left|\omega_{-i}-\bar{\omega}\right|<\gamma$, then $\lim _{n \rightarrow \infty} \omega_{n}=\bar{\omega}$. Also, $\bar{\omega}$ is said to be a global attractor if for every $\omega_{-v} \in(0, \infty)$ for $v=0,1, \cdots, k$, we have $\lim _{n \rightarrow \infty} \omega_{n}=\bar{\omega}$. On the other hand, $\bar{\omega}$ is said to be unstable if it is not locally stable.

Finally, Equation (4) is called permanent and bounded if there exists numbers $m$ and $M$ with $0<m<M<\infty$ such that for any initial conditions $\omega_{-v} \in(0, \infty)$ for $v=0,1, \cdots, k$ there exists a positive integer $N$ which depends on these initial conditions such that $m<\omega_{n}<M$ for all $n \geq N$.

The linearized equation of Equation (1) about the equilibrium point $\hat{\omega}$ is

$$
y_{n+1}=\sum_{i=0}^{k} p_{i} y_{n-i} .
$$

where

$$
p_{i}=\frac{\partial F}{\partial \omega_{n-i}}(\hat{\omega}, \hat{\omega}, \cdots, \hat{\omega}) .
$$

Theorem 1.1 [12] Assume that $p_{i} \in \mathbb{R}$ for $i=0,1, \cdots, k$. Then

$$
\left|p_{0}\right|+\left|p_{1}\right|+\cdots+\left|p_{k}\right|<1
$$

is a sufficient condition for the asymptotic stability of Equation(1).

\section{The Stability of Solutions}

The positive equilibrium point of Equation (1) is given by

$$
\hat{\omega}=a+\frac{b_{0}}{c_{1}+c_{2}}+\frac{b_{1}}{c_{3}+c_{4}} .
$$

Now, we define a function $f \in C\left((0, \infty)^{4},(0, \infty)\right)$ such that

$$
f(u, v, w, r)=a+\frac{b_{0} v}{c_{1} u+c_{2} v}+\frac{b_{1} r}{c_{3} w+c_{4} r} .
$$

Therefore, we find

$$
\begin{gathered}
\frac{\partial f}{\partial u}=-\frac{b_{0} c_{1} v}{\left(c_{1} u+c_{2} v\right)^{2}}, \\
\frac{\partial f}{\partial v}=\frac{b_{0} c_{1} u}{\left(c_{1} u+c_{2} v\right)^{2}}, \\
\frac{\partial f}{\partial w}=-\frac{b_{1} c_{3} r}{\left(c_{3} w+c_{4} r\right)^{2}}
\end{gathered}
$$

and

$$
\frac{\partial f}{\partial r}=\frac{b_{1} c_{3} w}{\left(c_{3} w+c_{4} r\right)^{2}} .
$$

Theorem 2.1. Assume that $\hat{\omega}$ be a +ve equilibrium point of Equation (1). If 


$$
b_{0} c_{1} \mu+b_{1} c_{3} \lambda<a \lambda \mu+b_{0} c_{2} \mu+b_{1} c_{4} \lambda .
$$

where $\lambda=\left(c_{1}+c_{2}\right)^{2}$ and $\mu=\left(c_{3}+c_{4}\right)^{2}$, then $\hat{\omega}$ is local stable.

Proof. From (7) to (10), we get

$$
\begin{gathered}
\frac{\partial f}{\partial u}(\hat{\omega}, \hat{\omega}, \hat{\omega}, \hat{\omega})=-\frac{b_{0} c_{1}}{\left(a+\frac{b_{0}}{c_{1}+c_{2}}+\frac{b_{1}}{c_{3}+c_{4}}\right)\left(c_{1}+c_{2}\right)^{2}}=p_{0}, \\
\frac{\partial f}{\partial v}(\hat{\omega}, \hat{\omega}, \hat{\omega}, \hat{\omega})=\frac{b_{0} c_{1}}{\left(a+\frac{b_{0}}{c_{1}+c_{2}}+\frac{b_{1}}{c_{3}+c_{4}}\right)\left(c_{1}+c_{2}\right)^{2}}=p_{1}, \\
\frac{\partial f}{\partial w}(\hat{\omega}, \hat{\omega}, \hat{\omega}, \hat{\omega})=-\frac{b_{1} c_{3}}{\left(a+\frac{b_{0}}{c_{1}+c_{2}}+\frac{b_{1}}{c_{3}+c_{4}}\right)\left(c_{1}+c_{2}\right)^{2}}=p_{2}
\end{gathered}
$$

and

$$
\frac{\partial f}{\partial r}(\hat{\omega}, \hat{\omega}, \hat{\omega}, \hat{\omega})=\frac{b_{1} c_{3}}{\left(a+\frac{b_{0}}{c_{1}+c_{2}}+\frac{b_{1}}{c_{3}+c_{4}}\right)\left(c_{1}+c_{2}\right)^{2}}=p_{3} .
$$

Thus, the linearized equation of (1), is

$$
y_{n+1}=p_{0} y_{n}+p_{1} y_{n-1}+p_{2} y_{n-2}+p_{3} y_{n-3} .
$$

From Theorem 1.1, we have that Equation (1) is locally stable if

$$
\begin{aligned}
& \left|-\frac{b_{0} c_{1}}{\left(a+\frac{b_{0}}{c_{1}+c_{2}}+\frac{b_{1}}{c_{3}+c_{4}}\right)\left(c_{1}+c_{2}\right)^{2}}\right|+\left|\frac{b_{0} c_{1}}{\left(a+\frac{b_{0}}{c_{1}+c_{2}}+\frac{b_{1}}{c_{3}+c_{4}}\right)\left(c_{1}+c_{2}\right)^{2}}\right| \\
& +\left|-\frac{b_{1} c_{3}}{\left(a+\frac{b_{0}}{c_{1}+c_{2}}+\frac{b_{1}}{c_{3}+c_{4}}\right)\left(c_{1}+c_{2}\right)^{2}}\right|+\left|\frac{b_{1} c_{3}}{\left(a+\frac{b_{0}}{c_{1}+c_{2}}+\frac{b_{1}}{c_{3}+c_{4}}\right)\left(c_{1}+c_{2}\right)^{2}}\right|<1,
\end{aligned}
$$

and hence,

$$
2 b_{0} c_{1}\left(c_{3}+c_{4}\right)^{2}+2 b_{1} c_{3}\left(c_{1}+c_{2}\right)^{2}<\left[a+\frac{b_{0}}{c_{1}+c_{2}}+\frac{b_{1}}{c_{3}+c_{4}}\right]\left(c_{1}+c_{2}\right)^{2}\left(c_{3}+c_{4}\right)^{2} .
$$

Then, we find

$$
\begin{aligned}
& b_{0} c_{1}\left(c_{3}+c_{4}\right)^{2}+b_{1} c_{3}\left(c_{1}+c_{2}\right)^{2} \\
& <a\left(c_{1}+c_{2}\right)^{2}\left(c_{3}+c_{4}\right)^{2}+b_{0} c_{2}\left(c_{3}+c_{4}\right)^{2}+b_{1} c_{4}\left(c_{1}+c_{2}\right)^{2},
\end{aligned}
$$

This completes the proof of Theorem 2.1.

Example 2.1. Figure 1 shows that Equation (1) has local stable solutions if $a=0.01, b_{0}=200, b_{1}=20, c_{1}=0.002, c_{2}=0.01, c_{3}=0.002$ and $c_{4}=0.001$.

Theorem 2.2. If $c_{1}=c_{2}=\alpha$ and $c_{3}=c_{4}=\beta$, then the equilibrium point $\hat{\omega}$ 


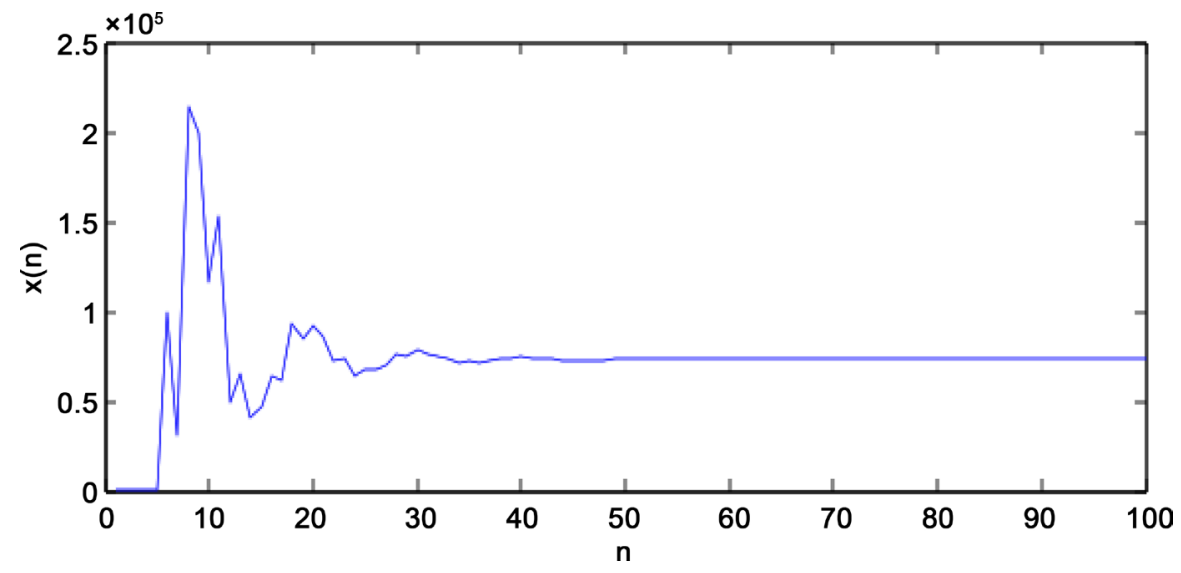

Figure 1. The stable solution corresponding to difference Equation (1).

Equation (1) is global attractor.

Proof. We consider the following function

$$
f(u, v ; w, r)=a+\frac{b_{0} v}{c_{1} u+c_{2} v}+\frac{b_{1} r}{c_{3} w+c_{4} r}
$$

Note that $f$ non-decreasing for $v, r$ and non-increasing for $u, w$. Let $(\psi, \Psi)$ a solution of the system

$$
\begin{aligned}
& \psi=f(\Psi, \psi, \Psi, \psi) \\
& \Psi=f(\psi, \Psi, \psi, \Psi)
\end{aligned}
$$

From (11), we have

$$
(\Psi-a)\left(c_{1} \psi+c_{2} \Psi\right)\left(c_{3} \psi+c_{4} \Psi\right)=b_{0} \Psi\left(c_{3} \psi+c_{4} \Psi\right)+b_{1} \Psi\left(c_{1} \psi+c_{2} \Psi\right)
$$

and

$$
(\psi-a)\left(c_{1} \Psi+c_{2} \psi\right)\left(c_{3} \Psi+c_{4} \psi\right)=b_{0} \psi\left(c_{3} \Psi+c_{4} \psi\right)+b_{1} \psi\left(c_{1} \Psi+c_{2} \psi\right)
$$

Thus, we get

$$
\begin{aligned}
& \alpha \beta(\Psi-a)(\Psi+\psi)=\Psi b_{0} \beta(\Psi+\psi)+\Psi b_{1} \alpha(\Psi+\psi), \\
& \alpha \beta(\Psi-a)(\Psi+\psi)=\psi b_{0} \beta(\Psi+\psi)+\Psi b_{1} \alpha(\Psi+\psi) .
\end{aligned}
$$

By subtracting (12) and (13), we have

$$
\left(b_{0} \beta+b_{1} \alpha\right)(\Psi-\psi)=0
$$

Since $b_{0} \beta+b_{1} \alpha \neq 0$, we get

$$
\Psi=\psi .
$$

This completes the proof of this Theorem.

\section{Periodic Solution of Period Two}

In this section, we investigate the existence of periodic solutions of Equation (1).

Theorem 3.1. Assume that $c_{1}=c_{3}=\alpha$ and $c_{2}=c_{4}=\beta$. Equation (1) has positive prime period-two solutions if

$$
\left(b_{0}+b_{1}\right)(\alpha-\beta)>a\left(\alpha^{2}+\beta^{2}\right)+2 a \alpha \beta .
$$


Proof. Suppose that there exists a prime period-two solution

$$
\cdots, \sigma, \rho, \sigma, \rho, \sigma, \rho, \cdots
$$

of Equation (1). We will prove that condition (14) holds. We see from Equation (1) that if $c_{1}=c_{3}=\alpha$ and $c_{2}=c_{4}=\beta$, then $\omega_{n}=\omega_{n-2}=\rho, \omega_{n+1}=\omega_{n-1}=\omega_{n-3}=\sigma$ and so,

$$
\sigma=a+\frac{b_{0} \sigma}{c_{1} \rho+c_{2} \sigma}+\frac{b_{1} \sigma}{c_{3} \rho+c_{4} \sigma} \text { and } \rho=a+\frac{b_{0} \rho}{c_{1} \sigma+c_{2} \rho}+\frac{b_{1} \rho}{c_{3} \sigma+c_{4} \rho} .
$$

Thus, we have

$$
\alpha \sigma \rho+\beta \sigma^{2}=a \alpha \rho+a \beta \sigma+b_{0} \sigma+b_{1} \sigma
$$

and

$$
\alpha \sigma \rho+\beta \rho^{2}=a \alpha \sigma+a \beta \rho+b_{0} \rho+b_{1} \rho .
$$

By subtracting (15) and (16), we have

$$
\beta\left(\sigma^{2}-\rho^{2}\right)=-a(\sigma-\rho)+a \beta(\sigma-\rho)+b_{0}(\sigma-\rho)+b_{1}(\sigma-\rho),
$$

then, we find

$$
\sigma+\rho=\frac{-\alpha a+\beta a+b_{0}+b_{1}}{\beta} .
$$

By combining (15) and (16), we have

$$
2 \alpha \sigma \rho+\beta\left(\sigma^{2}+\rho^{2}\right)=a \alpha(\sigma+\rho)+a \beta(\sigma+\rho)+b_{0}(\sigma+\rho)+b_{1}(\sigma+\rho)
$$

and so,

$$
\beta \sigma \rho(\alpha-\beta)=a \alpha\left(-a \alpha+a \beta+b_{0}+b_{1}\right)
$$

Since, $\sigma^{2}+\rho^{2}=(\sigma+\rho)^{2}-2 \sigma \rho$, we obtain

$$
\sigma \rho=\frac{a \alpha\left(-a \alpha+a \beta+b_{0}+b_{1}\right)}{\beta(\alpha-\beta)}
$$

Now,it is clear from (17) and (18) that $\sigma$ and $\rho$ are both two positive distinct roots of the quadratic equation

$$
u^{2}+(\sigma+\rho) u+\sigma \rho=0
$$

Hence, we obtain

$$
\frac{\left(-\alpha a+\beta a+b_{0}+b_{1}\right)^{2}}{\beta^{2}}-\frac{4 a \alpha\left(-a \alpha+a \beta+b_{0}+b_{1}\right)}{\beta(\alpha-\beta)}>0,
$$

which is equivalent to

$$
\left(b_{0}+b_{1}\right)(\alpha-\beta)>a\left(\alpha^{2}+\beta^{2}\right)+2 a \alpha \beta .
$$

This completes the proof of Theorem 3.1.

Example 3.1. Consider Equation (1) with $c_{1}=c_{4}=\beta=0.001$, $c_{3}=c_{4}=\alpha=0.002, a=0.01, b_{0}=200$ and $b_{1}=20000$. By Theorem 3.1, Equation (1) has prime period two solution (see Figure 2).

Example 3.2. Consider Equation (1) with $c_{1}=c_{4}=\beta=0.0003, c_{3}=c_{4}=$ 


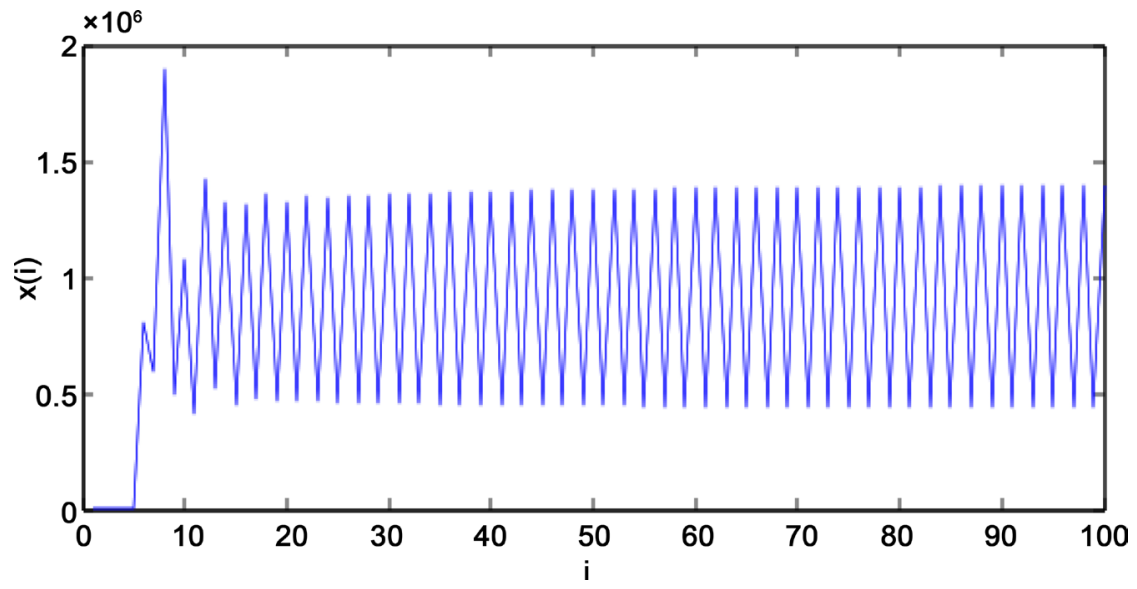

Figure 2. Prime period two solution of Equation (1).

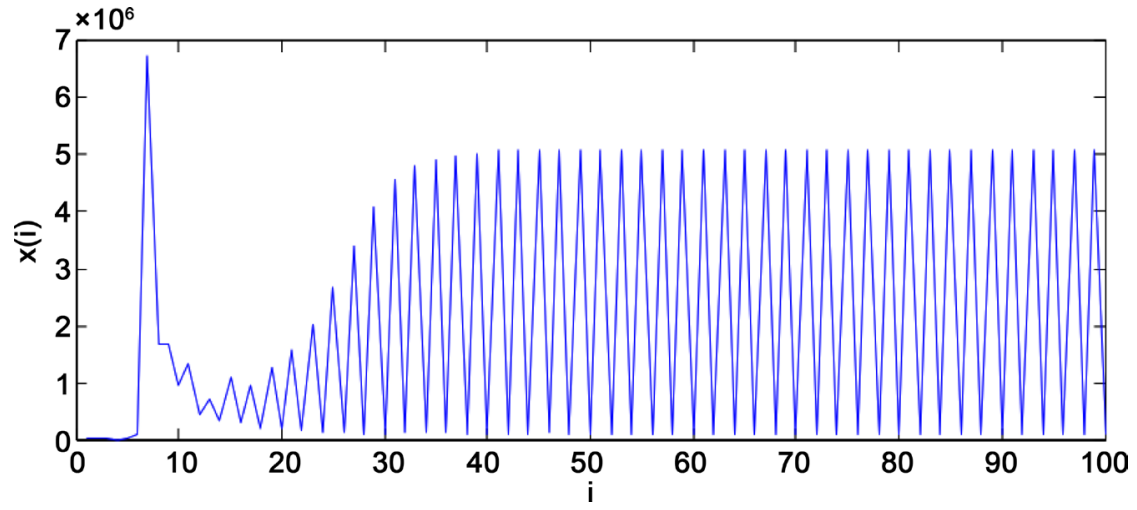

Figure 3. Prime period two corresponding to differences Equation (1).

$\alpha=0.005, a=0.8, b_{0}=500$ and $b_{1}=2000$. By Theorem 3.1, Equation (1) has prime period two solution (see Figure 3 ).

\section{Boundedness of the Solutions}

In this section, we study the characteristic task of boundedness of the positive solutions of Equation (1).

Theorem 4.1. Every solution of (1) is bounded and persists.

Proof. From Equation (1), we have

$$
\begin{aligned}
\omega_{n+1} & =a+\frac{b_{0} \omega_{n-1}}{c_{1} \omega_{n}+c_{2} \omega_{n-1}}+\frac{b_{1} \omega_{n-3}}{c_{3} \omega_{n-2}+c_{4} \omega_{n-3}} \\
& \leq a+\frac{b_{0} \omega_{n-1}}{c_{2} \omega_{n-1}}+\frac{b_{1} \omega_{n-3}}{c_{4} \omega_{n-3}} \\
& =a+\frac{b_{0}}{c_{2}}+\frac{b_{1}}{c_{4}}
\end{aligned}
$$

then,

$$
0<\omega_{n} \leq a+\frac{b_{0}}{c_{2}}+\frac{b_{1}}{c_{4}}=M
$$

Hence, the proof is completed. $\square$ 
Conclusion 1. This work is concerned with studying a dynamics and behavior of solutions of a new class of difference Equation (1). Our results extend and generalize to previous studies, for example, Equation (2) (if $\left.b_{1}=c_{1}=0\right)$ and Equation (3) (if $a=b_{1}=0$ ). Furthermore, we obtain the following results:

- The +ve equilibrium point $\hat{\omega}$ of equation (1) is local stable if $b_{0} c_{1} \mu+b_{1} c_{3} \lambda<a \lambda \mu+b_{0} c_{2} \mu+b_{1} c_{4} \lambda$, where $\lambda=\left(c_{1}+c_{2}\right)^{2}$ and $\mu=\left(c_{3}+c_{4}\right)^{2}$. Also, if $c_{1}=c_{2}$ and $c_{3}=c_{4}$, then $\hat{\omega}$ is global attractor.

- Equation (1) has a prime period-two solutions if $c_{1}=c_{3}=\alpha, c_{2}=c_{4}=\beta$ and $\left(b_{0}+b_{1}\right)(\alpha-\beta)>a\left(\alpha^{2}+\beta^{2}\right)+2 a \alpha \beta$.

- Every solution of $(1)$ is bounded and persists.

\section{Acknowledgements}

The authors are grateful to the editors and anonymous referees for a very thorough reading of the manuscript and for pointing out several inaccuracies.

\section{References}

[1] Abdelrahman, M.A.E. and Moaaz, O. (2017) Investigation of the New Class of the Nonlinear Rational Difference Equations. Fundamental Research and Development International, 7, 59-72.

[2] Abdelrahman, M.A.E. and Moaaz, O. (2018) On the New Class of the Nonlinear Difference Equations. Electronic Journal of Mathematical Analysis and Applications, 6, 117-125.

[3] Amleh, A.M., Grove, E.A., Georgiou, A. and Ladas, G. (1999) On the Recursive Sequence $\omega_{n+1}=\alpha+\frac{\omega_{n-1}}{\omega_{n}}$. Journal of Mathematical Analysis and Applications, 233, 790-798. https://doi.org/10.1006/jmaa.1999.6346

[4] Camouzis, E., DeVault, R. and Ladas, G. (2001) On the Recursive Sequence $\omega_{n+1}=-1+\omega_{n-1} / \omega_{n}$. Journal of Difference Equations and Applications, 7, 477-482.

[5] Elabbasy, E.M., EL-Metwally, H.A. and Elsayed, E.M. (2006) On the Difference Equation $\omega_{n+1}=a \omega_{n}-b \omega_{n} /\left(c \omega_{n}-d \omega_{n-1}\right)$. Advances in Difference Equations, 2006, $1-10$.

[6] Elaydi, S.N. (1996) An Introduction to Difference Equations, Undergraduate Texts in Mathematics, Springer, New York. https://doi.org/10.1007/978-1-4757-9168-6

[7] Elsayed, E.M. and El-Metwally, H.A. (2012) Qualitative Studies of Scalars and Systems of Difference Equations. Publisher LAP Lambert Academic.

[8] Elsayed, E.M. (2015) New Method to Obtain Periodic Solutions of Period Two and Three of a Rational Difference Equation. Nonlinear Dynamics, 79, 241-250. https://doi.org/10.1007/s11071-014-1660-2

[9] Elsayed, E.M. (2016) Dynamics and Behavior of a Higher Order Rational Difference Equation. Journal of Nonlinear Sciences and Applications, 9, 1463-1474.

[10] Elsayed, E.M. (2010) On the Global Attractivity and the Periodic Character of a Recursive Sequence. Opuscula Mathematica, 30, 431-446. https://doi.org/10.7494/OpMath.2010.30.4.431

[11] Grove, E.A. and Ladas, G. (2005) Periodicities in Nonlinear Difference Equations. Vol. 4, Chapman \& Hall/CRC. 
[12] Kulenovic, M.R.S. and Ladas, G. (2001) Dynamics of Second Order Rational Difference Equations with Open Problems and Conjectures. Chapman \& Hall/CRC. https://doi.org/10.1201/9781420035384

[13] El-Metwally, H., Ladas, G., Grove, E.A. and Voulov, H.D. (2001) On the Global Attractivity and the Periodic Character of Some Difference Equations. Journal of Difference Equations and Applications, 7, 837-850. https://doi.org/10.1080/10236190108808306

[14] Metwally, H.E., Grove, E.A. and Ladas, G. (2000) A Global Convergence Result with Applications to Periodic Solutions. Journal of Mathematical Analysis and Applications, 245, 161-170. https://doi.org/10.1006/jmaa.2000.6747

[15] Moaaz, O. and Abdelrahman, M.A.E. (2016) Behaviour of the New Class of the Rational Difference Equations. Journal of Mathematical Analysis and Applications, 4 129-138.

[16] Moaaz, O. (2017) On Comment on "New Method to Obtain Periodic Solutions of Period Two and Three of a Rational Difference Equation. Nonlinear Dynamics, 79, 241-250. https://doi.org/10.1007/s11071-016-3293-0

[17] Moaaz, O. and Abdelrahman, M.A.E. (2017) On the Class of the Rational Difference Equations. International Journal of Advances in Mathematics, 4, 46-55.

[18] Ocalan, O. (2014) Dynamics of the Difference Equation $\omega_{n+1}=p_{n}+\omega_{n-k} / \omega_{n}$ with a Period-Two Coefficient. Applied Mathematics and Computation, 228, 31-37.

[19] Saleh, M. and Aloqeili, M. (2005) On the Difference Equation $x_{n+1}=A+x_{n} / x_{n-k}$. Applied Mathematics and Computation, 171, 862-869.

[20] Sun, T. and Xi, H. (2007) On Convergence of the Solutions of the Difference Equation $\omega n+1=1+\frac{\omega n-1}{\omega n}$. Journal of Mathematical Analysis and Applications, 325, 1491-1494.

[21] Zhang, L., Zhang, G. and Liu, H. (2005) Periodicity and Attractivity for a Rational Recursive Sequence. Journal of Applied Mathematics and Computing, 19, 191-201. https://doi.org/10.1007/BF02935798 